\title{
ALIFE2 study: low-molecular-weight heparin for women with recurrent miscarriage and inherited thrombophilia - study protocol for a randomized controlled trial
}

Paulien G de Jong ${ }^{1 *}$, Siobhan Quenby ${ }^{2}$, Kitty WM Bloemenkamp ${ }^{3}$, Babette AM Braams-Lisman ${ }^{4}$, Jan Peter de Bruin ${ }^{5}$, Arri Coomarasamy ${ }^{6}$, Michele David ${ }^{7}$, Maria T DeSancho ${ }^{8}$, Olivier WH van der Heijden, Annemieke Hoek ${ }^{10}$, Barbara A Hutten ${ }^{11}$, Kristin Jochmans ${ }^{12}$, Carolien AM Koks ${ }^{13}$, Walter KH Kuchenbecker ${ }^{14}$, Ben Willem J Mol ${ }^{15}$, Helen L Torrance ${ }^{16}$, Hubertina CJ Scheepers ${ }^{17}$, Mary D Stephenson ${ }^{18}$, Harold R Verhoeve ${ }^{19}$, Jantien Visser ${ }^{20}$, Johanna IP de Vries ${ }^{21}$, Mariëtte Goddijn ${ }^{22 \dagger}$ and Saskia Middeldorp ${ }^{1 \dagger}$

\footnotetext{
Abstract

Background: A large number of studies have shown an association between inherited thrombophilia and recurrent miscarriage. It has been hypothesized that anticoagulant therapy might reduce the number of miscarriages and stillbirth in these women. In the absence of randomized controlled trials evaluating the efficacy of anticoagulant therapy in women with inherited thrombophilia and recurrent miscarriage, a randomized trial with adequate power that addresses this question is needed. The objective of the ALIFE2 study is therefore to evaluate the efficacy of low-molecular-weight heparin $(\mathrm{LMWH})$ in women with inherited thrombophilia and recurrent miscarriage, with live birth as the primary outcome.

Methods/Design: Randomized study of LMWH plus standard pregnancy surveillance versus standard pregnancy surveillance alone.

Study population: pregnant women of less than 7 weeks' gestation, and confirmed inherited thrombophilia with a history of 2 or more miscarriages or intra-uterine fetal deaths, or both.

Setting: multi-center study in centers from the Dutch Consortium of Fertility studies; centers outside the

Netherlands are currently preparing to participate.

Intervention: LMWH enoxaparin 40 mg subcutaneously once daily started prior to 7 weeks gestational age plus standard pregnancy surveillance or standard pregnancy surveillance alone.

Main study parameters/endpoints: the primary efficacy outcome is live birth. Secondary efficacy outcomes include adverse pregnancy outcomes, such as miscarriage, pre-eclampsia, syndrome of hemolysis, elevated liver enzymes and low platelets (HELLP syndrome), fetal growth restriction, placental abruption, premature delivery and congenital malformations.

Safety outcomes include bleeding episodes, thrombocytopenia and skin reactions.

(Continued on next page)
}

\footnotetext{
* Correspondence: p.g.dejong@amc.uva.nl

${ }^{\dagger}$ Equal contributors

'Department of Vascular Medicine, Academic Medical Center, University of

Amsterdam, Meibergdreef 9, 1105 AZ Amsterdam, the Netherlands

Full list of author information is available at the end of the article
} 
(Continued from previous page)

Discussion: After an initial period of slow recruitment, the recruitment rate for the study has increased. Improved awareness of the study and acknowledgement of the need for evidence are thought to be contributing to the improved recruitment rates. We aim to increase the number of recruiting centers in order to increase enrollment into the ALIFE2 study.

The study website can be accessed via www.ALIFE2study.org.

Trial registration: The ALIFE2 study was registered on 19 March 2012 under registration number NTR3361

Keywords: Recurrent miscarriage, Pregnancy complications, Fetal death, Thrombophilia, Anticoagulants, Aspirin, Low-molecular-weight-heparin

\section{Background}

In all clinically recognized pregnancies, a single spontaneous miscarriage occurs in 14 to $19 \%$ of patients, and 1 to $5 \%$ of women experience 2 or more miscarriages (recurrent miscarriage) [1,2]. After comprehensive investigation, a risk factor for recurrent miscarriage is identified in less than half of couples [3]. Many studies have confirmed a relationship between inherited thrombophilia and miscarriage and other adverse pregnancy outcomes $[4,5]$. The role of thrombophilia in recurrent miscarriage can be explained partially by the concept of thrombosis of the (microvasculature of the) placenta, and partially through inhibition of extra-villous trophoblast differentiation [6]. Therefore, anticoagulants are considered a possible therapy for women with recurrent miscarriage and inherited thrombophilia. In a recent Cochrane systematic review and meta-analysis we reviewed the trial evidence on the effects of antithrombotic therapy and showed that no firm trial data are available for these women [7].

Previously, we performed a randomized, placebo controlled study (ALIFE study), investigating whether aspirin combined with low-molecular-weight heparin (LMWH) or aspirin alone as compared with placebo would improve the live birth rate among 364 women with unexplained recurrent miscarriage $(<20$ weeks' gestation) [8]. We found that neither aspirin combined with nadroparin (relative risk (RR) for live birth 0.96, 95\% confidence interval (CI) 0.76 to 1.19 ) nor aspirin alone (RR for live birth $0.89,95 \%$ CI 0.71 to 1.13 ) improved the chance of a live birth in women with a history of unexplained recurrent miscarriage. In addition, no statistically significant benefits were found for women with inherited thrombophilia, although the study was not powered to assess this effect. The SPIN-study, another randomized controlled trial, assessed whether enoxaparin and aspirin reduced the rate of miscarriage compared to intensive pregnancy surveillance alone in 294 women with a history of 2 or more consecutive previous miscarriages $(<24$ weeks' gestation) [9]. Results showed $22 \%$ miscarriage in participants receiving enoxaparin and aspirin, compared with $20 \%$ miscarriage in subjects receiving intensive surveillance alone (odds ratio 0.91,
95\% confidence interval 0.52 to 1.59). The Habenox study also found no beneficial effect of LMWH (with aspirin or placebo) compared to aspirin in women with or without thrombophilia and recurrent miscarriage [10]. Neither individually, nor combined in meta-analysis, was any of these studies sufficiently powered to demonstrate an effect of pharmacological therapy in the subgroup of women with inherited thrombophilia [7]. For women with the antiphospholipid syndrome (APS), the use of heparin or LMWH combined with low-dose aspirin is an effective treatment for recurrent miscarriage, [11-13] although not demonstrated in all performed trials $[14,15]$. For inherited forms of thrombophilia, this effect has not profoundly been studied yet. Very recently, results of the TIPPS study showed that dalteparin did not reduce the incidence of a composite outcome (severe or early-onset pre-eclampsia, small-for-gestational-age infant, pregnancy loss, or venous thromboembolism (VTE)) in pregnant women with thrombophilia at increased risk of VTE or with previous placenta-mediated pregnancy complications [16]. However, again in this study, the subgroup of women with recurrent miscarriage and inherited thrombophilia was too small to draw conclusions of a potential effect on the outcome live birth.

The association between inherited thrombophilia and recurrent miscarriage together with the potential beneficial effects of anticoagulant therapy in women with acquired thrombophilia and recurrent miscarriage have led some physicians to prescribe LMWH to women with recurrent miscarriage and inherited thrombophilia. While this practice may be supported by a plausible hypothesis, a beneficial effect of LMWH in these women still needs to be demonstrated. Gynecologists and hematologists, who expressed their support in the design phase of the study, and who are currently contributing to recruitment, recognize the urgent need for a trial such as ALIFE2 internationally.

The results of the ALIFE2 study will clarify the need to screen for inherited thrombophilia in women with recurrent miscarriage. Current guidelines advise differently on whether or not to test for inherited thrombophilia in these women [17-22]. The efficacy of LMWH in women 
with recurrent miscarriage and inherited thrombophilia has never been tested in a randomized controlled trial. Thus, if the results of the ALIFE2 study show that LMWH increases live birth in women with recurrent miscarriage and inherited thrombophilia, screening for inherited thrombophilia in this setting may be justified. Conversely, if no evidence of a benefit is found, the use of LMWH will not be justified and screening for inherited thrombophilia will not be indicated. This will decrease costs of inappropriate screening, and reduce the burden of anticoagulant treatment in pregnant women.

\section{Methods/Design}

\section{Design of the study}

The primary objective of this study is to evaluate the efficacy of LMWH in women with inherited thrombophilia and recurrent miscarriage and/or intra-uterine fetal death $(\geq 2)$. The primary outcome is live birth.

Secondary objectives included efficacy and safety objectives:

Efficacy

- To evaluate other possible effects of LMWH on adverse pregnancy outcome other than miscarriage (e.g., preeclampsia, intra-uterine growth restriction, HELLP syndrome (syndrome of hemolysis, elevated liver enzymes and low platelets) placental abruption, premature delivery, congenital malformations, VTE)

\section{Safety}

- To evaluate the safety of LMWH in women with recurrent miscarriage with inherited thrombophilia by recording complications such as bleeding, thrombocytopenia and (allergic) skin reactions

All pre-specified outcomes are listed in Table 1. Criteria for the classification of bleeding are listed in Table 2.

Other study variables include concomitant use of antithrombotic drugs (such as aspirin or non-steroidal anti-inflammatory drugs (NSAIDs), maternal age, multiple gestation, number of preceding miscarriages, maternal ethnicity, partner's ethnicity, maternal weight, height, and body mass index, past obstetric history, medication, smoking, alcohol consumption, allergies, family history of VTE and of miscarriage and/or intrauterine fetal death.

The study is designed as a multi-center randomized intervention study (Figure 1). Pregnant women with a history of recurrent miscarriage (two or more miscarriages or intra-uterine fetal deaths, not necessarily consecutive), and confirmed inherited thrombophilia are randomized to one of two groups. Women in the

\section{Table 1 Outcomes}

\begin{tabular}{|c|c|}
\hline $\begin{array}{l}\text { Primary efficacy } \\
\text { outcome }\end{array}$ & Live birth (defined as birth of a living child) \\
\hline $\begin{array}{l}\text { Principal safety } \\
\text { outcome }\end{array}$ & $\begin{array}{l}\text { Clinically relevant bleeding (that is major bleeding } \\
\text { and clinically relevant non-major bleeding) }\end{array}$ \\
\hline \multicolumn{2}{|l|}{$\begin{array}{l}\text { Secondary study } \\
\text { outcomes }\end{array}$} \\
\hline \multirow[t]{9}{*}{ Efficacy } & Ongoing pregnancy beyond 12 weeks' gestation \\
\hline & Preeclampsiaab \\
\hline & HELLP syndrome ${ }^{a c}$ \\
\hline & Intrauterine growth restriction ${ }^{\text {ad }}$ \\
\hline & Placental abruption ae \\
\hline & Premature birth $^{\text {af }}$ \\
\hline & Intra-uterine fetal death ${ }^{\mathrm{a}}$ \\
\hline & Major congenital anomalies ${ }^{\mathrm{ag}}$ \\
\hline & $\begin{array}{l}\text { Composite of confirmed deep vein thrombosis } \\
\text { and confirmed pulmonary embolism }\end{array}$ \\
\hline \multirow[t]{5}{*}{ Safety } & $\begin{array}{l}\text { Post-partum bleeding and severe post-partum } \\
\text { bleeding }\end{array}$ \\
\hline & Major bleeding \\
\hline & Clinically relevant non-major bleeding \\
\hline & $\begin{array}{l}\text { Minor bleeding, including increased tendency } \\
\text { to bruising not fulfilling the criteria for clinically } \\
\text { relevant non-major bleeding }\end{array}$ \\
\hline & $\begin{array}{l}\text { Heparin-induced thrombocytopenia } \\
\text { (defined according to ACCP criteria [24]) }\end{array}$ \\
\hline
\end{tabular}

Allergic reactions (redness or itching) localized at the injection site of $\mathrm{LMWH}$

Type 1 allergy: e.g. generalized symptoms including anaphylaxis 
Table 2 Criteria for major, clinically relevant non-major and minor bleeding

Major bleeding

Clinically relevant non-major bleeding
- Associated with a fall in hemoglobin of $2 \mathrm{~g} / \mathrm{dL}$ or more

- Leading to a transfusion of two or more units of packed red blood cells or whole blood

- Occurring in a critical site: intracranial, intra-spinal, intra-ocular, pericardial, intra-articular, intramuscular with compartment syndrome, retro-peritoneal

- Contributing to death

- Any bleeding compromising hemodynamics

- Any bleeding leading to hospitalization

- Subcutaneous hematoma larger than $25 \mathrm{~cm}^{2}$, or $100 \mathrm{~cm}^{2}$ if there was a traumatic cause

- Intramuscular hematoma documented by ultrasonography

- Epistaxis lasting > 5 minutes, repetitive (defined as two or more episodes of bleeding more extensive than spots on a handkerchief within 24 hours), or leading to an intervention (e.g..$\prime$ packing or electrocoagulation)

- Gingival bleeding occurring spontaneously (not related to eating, flossing or tooth brushing) or lasting $>5$ minutes

- Macroscopic spontaneous hematuria or hematuria that lasted $>24$ hours after instrumentation (e.g., catheter placement or surgery) of the urogenital tract

- Macroscopic gastrointestinal hemorrhage, including at least one episode of melena or hematemesis, with positive results on a fecal occult-blood test

- Hemoptysis, if more than a few speckles in the sputum and not occurring within the context of pulmonary embolism

- Any other bleeding type considered to have clinical consequences for a patient such as medical intervention, the need for unscheduled contact (visit or telephone call) with a physician, or temporary cessation of a study drug or associated with pain or impairment of daily life activities

Minor bleeding bleeding or post-partum bleeding.

intervention group inject LMWH once daily in addition to standard pregnancy surveillance. Women in the control group receive standard pregnancy surveillance. Since blinding of patients or investigators is deemed unfeasible due to the nature of the intervention, an adjudication committee, whose members are blinded for the intervention, will assess primary and secondary outcome measures. End of follow-up is at 6 weeks after delivery or miscarriage. Members of the blinded adjudication committee will be Prof. Dr. MH Prins, MD, epidemiologist and Dr. WM Ankum, gynecologist.

The study intervention is LMWH at a dose that has been widely used in pregnant patients, and that is comparable with prophylaxis of VTE after orthopedic surgery, and is administered once daily, subcutaneously. The rationale for this dose is that it is high enough to produce an effect (that is higher than the standard prophylaxis dose of $20 \mathrm{mg}$ ), but not as high as the therapeutic VTE dose, to minimize the bleeding risks. Furthermore, there is great experience with this specific anticoagulant in obstetrics and previous studies and its safety profile is well established. Women are instructed to inject themselves daily in either the upper leg or abdomen.

The recommended intervention is:

- Clexane (enoxaparin, Sanofi-Aventis Netherlands B.V., Kampenringweg 45 E, 2803 PE GOUDA, the
Netherlands) 40 mg LMWH, administered subcutaneously, $100 \mathrm{mg} / \mathrm{mL}, 0.4 \mathrm{~mL}$ syringe.

If the recommended intervention is unavailable (e.g.,, for practical or financial reasons in different countries), another type of LMWH in a dosage equivalent to enoxaparin $40 \mathrm{mg}$ can be chosen from the list below:

- Fraxiparin (nadroparin, GlaxoSmithKline bv, 980 Great West Road, Brentford, Middlesex, United Kingdom) 3,800 IU anti-Xa LMWH, 9,500 IU anti$\mathrm{Xa} / \mathrm{mL}, 0.4 \mathrm{~mL}$ syringe.

- Fragmin (dalteparin, Pfizer bv, 235 East 42nd Street, NY, NY 10017, USA) 5,000 IU LMWH, 25,000 IU/ $\mathrm{mL}, 0.2 \mathrm{~mL}$ syringe.

- Innohep (tinzaparin, Leo Pharma bv, Industriparken 55, DK-2750 Ballerup, Denmark) 4,500 IU LMWH, 4,500 IU syringe.

Doses will not be adjusted to body weight. In case none of the LMWH preparations is available, any other locally available $\mathrm{LMWH}$, in a corresponding dosage and approved by the Trial Management Team (headed by principal investigators S Middeldorp and M Goddijn) is allowed.

Apart from the assigned study medication, women are strongly discouraged from using antithrombotic or other medications that affect hemostasis, including NSAIDs. 


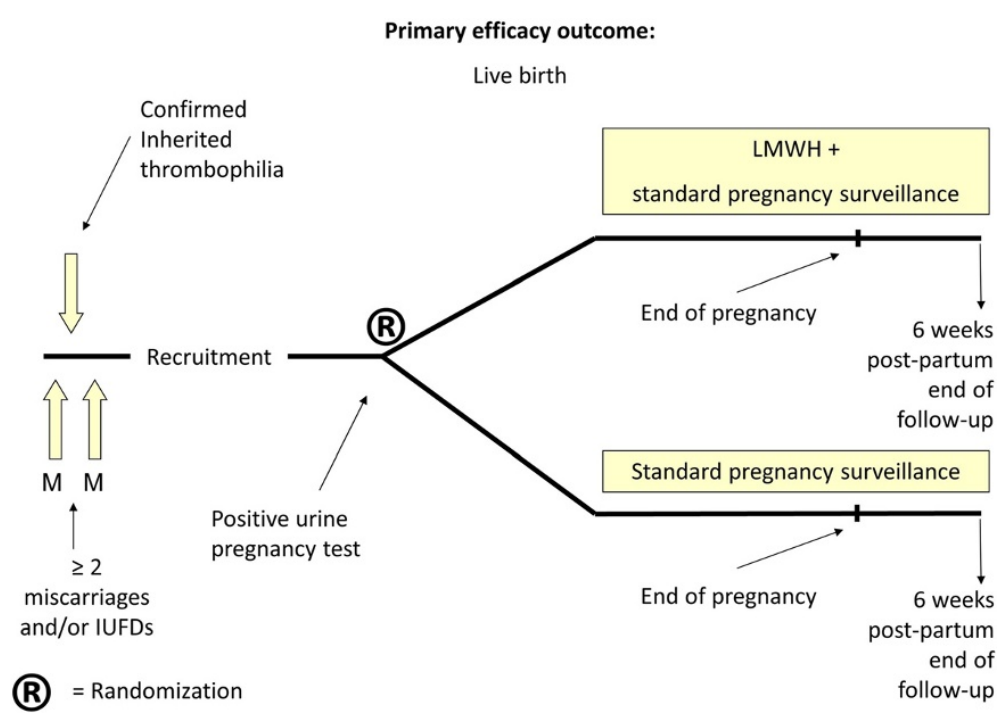

Figure 1 Flowchart of the ALIFE2 study.

Aspirin at a low dose ( $\leq 100 \mathrm{mg}$ daily) to lower the risk for recurrent preeclampsia (at the discretion of the treating physician) is allowed, and is recorded. All women are encouraged to take folic acid $400 \mu \mathrm{g}$ daily, starting before conception and continuing until 8 weeks after conception, as routine prophylaxis for neural tube defects.

The study will be conducted in several international centers, both tertiary and non-tertiary. A list of participating centers and countries is available from the sponsor.

\section{Ethics}

The study was approved by the Institutional Review Board (IRB) of the Academic Medical Center (IRB registration number METC_2012_173) and is conducted according to the principles of the World Medical Association (WMA) Declaration of Helsinki - Ethical Principles for Medical Research Involving Human Subjects Version Seoul, South Korea, October 2008, with Note of Clarification on Paragraph 29 added by the WMA General Assembly, Washington 2002; Note of Clarification on Paragraph 30 added by the WMA General Assembly, Tokyo 2004 and in accordance with the Dutch Medical Research Involving Human Subjects Act (WMO) and other guidelines, regulations and Acts.

Recruitment in individual centers will be commenced after local approval is obtained.

\section{Study procedures}

Women, aged 18 to 42 at randomization, with a history of 2 or more miscarriages and/or intra-uterine fetal deaths with confirmed inherited thrombophilia, who are actively trying to conceive or are less than 7 weeks pregnant are recruited for the study. They are informed of the study by their treating physician and receive written study information. Women are instructed to perform a urine pregnancy test as soon as their menstrual periods are delayed or a pregnancy is suspected. Furthermore, they are contacted by telephone at 3-month intervals until they are pregnant. Once a urine test confirms pregnancy and all in- and exclusion criteria are met (Table 3), including signing of informed consent (see Additional file 1), they are randomized to either the intervention arm or the standard pregnancy surveillance arm of the study. Informed consent will be obtained by GoodClinical-Practice-trained study personnel with use of the review-board approved consent form. Women will only be randomized in the study after informed consent is obtained.

Randomization is performed centrally, online via a secure Internet facility in a 1:1 ratio by the TENALEA Clinical Trial Data Management System using randomly permuted blocks with maximum block size of 6 within strata formed by maternal age ( $<36$ or $\geq 36$ years), number of miscarriages $(2$ or $\geq 3)$ and center type (tertiary or non-tertiary). Both the including physician and patient are concealed for allocation.

Women who are randomized to intervention arm start with LMWH immediately. They undergo a blood test twice ( $2.7 \mathrm{~mL}$ at baseline and $2.7 \mathrm{~mL}$ after 7 to 10 days) to check for heparin-induced thrombocytopenia and are instructed to discontinue LMWH when they experience the first signs of labor. In case of a planned delivery or cesarean section, LMWH should be discontinued according to local policy, but at least 12 hours prior to neuraxial anesthesia or cesarean section. Randomized women will be contacted at 12 and 24 weeks' gestation and 6 weeks post-partum to collect outcome data and 
Table 3 Inclusion and exclusion criteria

\begin{tabular}{|c|c|}
\hline \multirow[t]{10}{*}{ Inclusion criteria } & $\begin{array}{l}\text { - Women with recurrent miscarriage and/or intra-uterine fetal deaths (that is } \geq \text { two miscarriages of intra-uterine } \\
\text { fetal deaths, irrespective of gestational age) }\end{array}$ \\
\hline & - Confirmed inherited thrombophilia ${ }^{a}$ \\
\hline & - factor $V$ Leiden mutation \\
\hline & - prothrombin gene mutation (G20210A) \\
\hline & - protein S deficiency \\
\hline & - protein C deficiency \\
\hline & - antithrombin deficiency \\
\hline & - Pregnancy confirmed by urine pregnancy test \\
\hline & - Age 18 to 42 years at randomization \\
\hline & - Willing and able to give informed consent \\
\hline \multirow[t]{5}{*}{ Exclusion criteria } & - Duration of current pregnancy $\geq 7$ weeks, based on first day of last menstruation \\
\hline & $\begin{array}{l}\text { - Indication for anticoagulant treatment during pregnancy (e.g., prosthetic heart valves, a history of venous } \\
\text { thromboembolism or antiphospholipid syndrome) }\end{array}$ \\
\hline & $\begin{array}{l}\text { - Contraindications to LMWH (previous heparin-induced thrombocytopenia, active bleeding or renal insufficiency } \\
\text { with creatinine clearance of }<30 \mathrm{ml} / \text { minute) }\end{array}$ \\
\hline & - Known allergy to at least three different LMWH preparations \\
\hline & - Previous inclusion in the ALIFE2 study (for another pregnancy) \\
\hline
\end{tabular}

${ }^{a}$ Protein $\mathrm{S}$, protein $\mathrm{C}$ and antithrombin deficiencies need to be confirmed by two tests, performed on two separate occasions and not during anticoagulant therapy. Protein $\mathrm{S}$ tests should not be performed during pregnancy or in the 6-week post-partum period since spuriously low levels may then be observed.

data on adverse events. No additional study visits are planned.

Women in both study arms will receive standard pregnancy surveillance. Data from routine obstetric visits is recorded in a case record form (CRF) for analysis, including medical history and family history. Additionally, maternal bleeding episodes, thrombotic signs, and possible reactions to study medication are monitored. Women are instructed to contact the study center immediately when bleeding episodes occur or when developing (possibly heparin- induced) thrombocytopenia.

In case of a (serious) adverse event, emergency medication is administered by treating physicians according to local policy; e.g., in case of a major bleeding a patient may receive blood transfusions and/or antidote (e.g., protamine sulfate).

In case of serious type IV delayed hypersensitivity skin reactions to $\mathrm{LMWH}$, an alternative form of LMWH can be prescribed. In case of type I allergy, LMWH must be discontinued.

The CRF can be downloaded from the study website www.ALIFE2study.org. Data handling will be coded, with the patient code only available to the local investigator and the research nurse working in the local center. Data recorded in the CRFs will be collected in an electronic CRF, accessible via the study website.

Women withdrawn from treatment will be asked to continue follow-up until the end of study (that is 6 weeks after delivery or miscarriage).
Important protocol modifications will be communicated to all relevant parties (e.g.,.. investigators, trial nurses, review boards, trial participants, trial registries and journals) if indicated.

The study sponsor is the Academic Medical Center, University of Amsterdam, Meibergdreef 9, 1105 AZ, Amsterdam, the Netherlands. The currently approved study protocol is version 4.0, dated 18 August 2014. The full study protocol can be accessed via the study website (www.ALIFE2study.org).

Recruitment is currently taking place in the Netherlands and Belgium and is planned for the United Kingdom, the United States of America, Canada, and Australia and is open to more countries.

\section{Statistical analyses}

Baseline data and outcome data will be summarized separately. For continuous variables, we will examine the distribution of the observations and, if normally distributed, we will then summarize them as means with standard deviations (SDs). If they are not normally distributed, then medians and inter-quartile ranges (IRQs) will be reported. For dichotomous data, we will provide proportions (or percentages).

Differences in dichotomous outcomes between the two treatment arms will be analyzed using the chi-square test. For continuous outcomes we will use the independent $t$-test if the observations in each study arm are normally distributed, and if non-normally distributed, the 
Mann-Whitney $U$-test will be employed. For all outcome measures, we will calculate $95 \%$ CIs around point estimates.

The exploration of differential or subgroup effects of LMWH will be assessed in a priori-defined prognostic groups: age (continuous and dichotomized), number of previous miscarriages (two, or three or more), previous live birth (yes/no), type of inherited thrombophilia (factor V Leiden mutation, Prothrombin gene G20210A mutation PTM, antithrombin deficiency, protein C deficiency and protein $\mathrm{S}$ deficiency).

For issues such as loss to follow-up, missing data, and protocol violations, we will attempt sensitivity ('worstcase and best-case scenarios') analyses to explore the effect on the study findings. As a secondary analysis, we will adjust for missing data using imputation techniques to explore the effects of such imputations on the study findings.

Efficacy analyses will be based on the intention-totreat (ITT) principle. A $P$-value of less than 0.05 will be considered statistically significant. All statistical analyses will be performed using the SPSS package (SPSS Inc., Chicago, IL, USA).

\section{Sample size calculation}

In the previous ALIFE study [8], the occurrence of live birth in the subgroup of women who had an inherited thrombophilia and became pregnant was $60 \%$ in those randomized to placebo. Based on this information, a conservative sample size assuming a live birth of $55 \%$ in the control group is calculated. In order to detect an absolute risk difference of $15 \%$, with a power of $80 \%$ and a 2 -sided confidence level of $95 \%$, the sample size required for the study is $\mathrm{n}=332$, with 166 women in each arm. Taking a potential loss to follow-up and exclusion from the study (due to ectopic pregnancy for example) into consideration, we aim to recruit $20 \%$ more women $(\mathrm{n}=399)$.

The absolute risk difference of $15 \%$ was defined following consultations amongst health care providers and patients. Since we acknowledge that from a patient's perspective, a $10 \%$ absolute risk difference is still clinically relevant, we also plan to perform a conditional interim analysis to adjust the sample size. When approximately $95 \%$ of inclusion is reached, the adjudication committee will analyze efficacy outcome, blinded for allocation. If the estimated difference in live birth between the 2 groups is below $10 \%$ or above $15 \%$, the study will continue as planned and will be completed with 399 enrolled women. However, if the estimated difference in live birth is between $10 \%$ and $15 \%$, we will explore (financial) means and evaluate if continued recruitment until a total sample size of 776 women is feasible so that the study is sufficiently powered to assess this effect. For this interim analysis we will use a total 2-sided significance test with the O'Brien-Fleming alpha spending function and a type I error rate of $5 \%$. Evaluable women are all women included (randomized) in the study.

In the Netherlands we aim to recruit 200 women; therefore, approximately 200 women will be recruited in other countries. This will be pragmatically adjusted depending on recruitment status.

\section{Data Monitoring Committee (DMC)}

A Data Monitoring Committee is installed for the ALIFE2 study. A DMC charter is supplementary to this protocol and available from the sponsor. Monitoring will be performed in collaboration with the Academic Medical Center Clinical Research Unit (CRU). A monitoring plan was drafted accordingly and is available with the sponsor. Members of the DMC are Prof. Dr. HR Büller, AMC, Amsterdam (chair); Dr. WM Ankum, gynecologist, AMC, Amsterdam and Prof. Dr. M Prins, statistician, Maastricht University Medical Center, Maastricht.

The final trial dataset will be accessible by the lead investigator and statistical analysis team at the Academic Medical Center CRU. No contractual arrangements are in place that limit such access for investigators

\section{Adverse events}

LMWH is registered and recommended for a wide variety of indications. Also to pregnant women, LMWH is prescribed for several indications and in higher dosages than is used in the ALIFE2 study. Therefore, the spectrum of adverse events is well documented in regular patients as well as in pregnant women. For this reason, not all undesirable experiences occurring to women are recorded, but only adverse events that are suspected to be related to study medication. These adverse events include: clinically relevant bleeding (major or clinically relevant non-major bleeding) and any bleeding (hematoma, epistaxis, bleeding of gums, vaginal blood loss, hematuria or any other form of bleeding), heparin-induced thrombocytopenia, skin reaction to injection (e.g.,, itching, swelling) and (type 1) allergic reactions. When hematomas are related to venipuncture, only hematomas larger than $10 \times 10 \mathrm{~cm}$ are reported.

For all events recorded, the nature and severity will be assessed. A serious adverse event (SAE) is any untoward medical occurrence or effect that at any dose that:

- results in death

- is life-threatening (at the time of the event)

- requires hospitalization or prolongation of existing inpatient hospitalization

- results in persistent or significant disability or incapacity

- is a congenital anomaly or birth defect 
- is a new event of the study likely to affect the safety of the subjects, such as an unexpected outcome of an adverse reaction, lack of efficacy of an investigational medicinal product used for the treatment of a life-threatening disease, major safety finding from a newly completed animal study, and so on

SAEs that result in death or are life-threatening should be reported immediately. The reporting will occur not later than 7 days after the responsible investigator has first knowledge of the adverse reaction. This is for a preliminary report with another 8 days for completion of the report. The study efficacy outcome 'bleeding' will not be reported as SAE. A standard practice of hospitalization (e.g., for delivery) will not be considered as a prolonged hospitalization and should not be reported as an SAE. However, if this transfer is part of treatment of a medical complication, it should be considered prolonged hospitalization and the event should be reported as a SAE. Clinically anticipated events, such as bleeding, are exempted from the expedited reporting to regulatory authorities of suspected adverse reactions that are both serious and unexpected. These clinically anticipated events are periodically reviewed by the DMC in an unblinded manner to ensure prompt identification of any clinically concerning safety issues.

\section{Public disclosure and publication policy}

A writing committee chaired by the principal investigators will be put in place as the trial progresses. The composition of the committee will reflect the contribution of investigators to various aspects of the trial, including but not solely the conception and design, acquiring of funding, country coordination and recruitment of patients. This committee will write the study report, and the report will include list of centers and investigators that contributed patients to the study. There are no publications restrictions by the sponsor of the study.

Furthermore, trial results will be posted on the study website and communicated through patients via patient organization websites.

\section{Compensation for injury}

The sponsor/investigator in the Netherlands has a liability insurance that is in accordance with article 7, subsection 6 of the WMO.

The sponsor (also) has an insurance which is in accordance with the legal requirements in the Netherlands (Article $7 \mathrm{WMO}$ and the Measure regarding Compulsory Insurance for Clinical Research in Humans of 23 June 2003). This insurance provides cover for damage to research subjects through injury or death caused by the study:

1. $€ 450,000$ - for death or injury for each subject who participates in the research;

2. €3,500,000 - for death or injury for all subjects who participate in the research;

3. $€ 5,000,000$ - for the total damage incurred by the organization for all damage disclosed by scientific research for the sponsor as 'verrichter' in the meaning of said Act in each year of insurance coverage.

The insurance applies to the damage that becomes apparent during the study or within 4 years after the end of the study and is only applicable to patients included in the Netherlands.

\section{Discussion}

The choice for the intervention with LMWH in the ALIFE2 study was based on the following considerations. In the previous ALIFE study, LMWH was administered in combination with aspirin and compared with aspirin alone and to no-intervention (placebo for aspirin) in women with recurrent miscarriage of unknown cause [8]. Since there are no solid data supporting an effect of aspirin in women with recurrent miscarriage, with or without inherited thrombophilia, we chose to use LMWH as intervention, without aspirin as additional intervention in the ALIFE2 study, to exclude a possible deleterious effect of aspirin.

The dose of LMWH is higher than low-prophylactic dosages, in order not to miss a beneficial effect of LMWH on live birth. However, because results from the Live-Enox study showed no difference in live birth in women randomized to $40 \mathrm{mg}$ enoxaparin or $80 \mathrm{mg}$ enoxaparin, we chose to use the dosage of $40 \mathrm{mg}$ enoxaparin to reduce the bleeding risk [23].

A timely execution of the trial is impeded because of two reasons. Firstly, the assumed efficacy of LMWH triggers physicians to give LMWH the benefit of the doubt. They offer the treatment despite a lack of its evidence; especially in poignant cases as for example women with a history of multiple miscarriages. Eligible women will thus be reluctant to be randomized, facing a $50 \%$ chance of no-treatment, when they can also approach physicians who are willing to prescribe them LMWH outside the context of the study. This hurdle can be overcome, provided both physicians and patients are well informed. Physicians who are aware of the lack of evidence for LMWH and the non-negligible bleeding risk associated with this treatment clearly understand the need for the study. They are more willing to contribute to recruitment and comply with guidelines advising not to treat in absence of evidence for treatment. A 
second reason for delay, potentially specific to the Dutch situation, is that eligible women for the study remain unidentified because screening for inherited thrombophilia is not standard practice. A complete thrombophilia screen is expensive and as currently no proven effective treatment for women with recurrent miscarriage and inherited thrombophilia is available, testing for inherited thrombophilia (solely for the indication or recurrent miscarriage) is not always performed. Nonetheless, many women have already been tested for thrombophilia because of other reasons such as a positive family history of VTE. Moreover, as screening for inherited thrombophilia may provide an explanation for recurrent miscarriage, some physicians perform screening not to identify a treatable condition but to provide couples with this explanation. In this way, even physicians who do not incorporate thrombophilia screening in their standard practice can contribute to recruitment, identifying these women.

\section{Trial status}

The study commenced recruiting in January 2013 in the Netherlands, with at present 11 participating centers and 2 additional centers for which ethics approval is expected shortly. Participation of non-Dutch centers was initiated by the University Hospital of Brussels in Belgium, which is expected to be followed closely by centers in the United Kingdom, the United States, Canada and Sweden.

At the time of writing 23 women have been randomized in the study. An initially slow recruitment is currently catching up to a rate of approximately 2 inclusions per month in the Netherlands, expected to increase to 4 per month with continuing expansion of the number of participating centers and increasing awareness of the study. Confirmed participation of centers abroad and especially dedicated recurrent miscarriage clinics in the United Kingdom is expected to further increase this rate to an eventual inclusion rate of 14 women per month.

As with most investigator-initiated multi-center trials, several hurdles, such as an administrative workload, (international) legislation and applications for approval in participating centers, and initial slow recruitment, had to be overcome to get the trial running. However, with these hurdles overcome, the trial now seems to have reached a new phase, with a steadily increasing recruitment rate, towards completion of the trial.

\section{Endnote}

The study website can be accessed via www.ALIFE2study.org. The study is still open for additional participating centers. If you consider participation in the ALIFE2 study, please contact the study authors.

\section{Additional file}

Additional file 1: Patient information and informed consent form.

\section{Abbreviations}

ACCP: American College of Chest Physicians; APS: antiphospholipid syndrome; CRF: case record form; CRU: Clinical Research Unit; CT: computed tomography; DMC: Data Monitoring Committee; HELLP syndrome: syndrome of hemolysis, elevated liver enzymes and low platelets; IRB: Institutional Review Board; IRQ: inter-quartile range; ITT: intention-to-treat; LMWH: lowmolecular-weight heparin; NSAIDs: non-steroidal anti-inflammatory drugs; RR: relative risk; SAE: serious adverse event; VTE: venous thromboembolism; WMA: World Medical Association; WMO: Dutch Medical Research Involving Human Subjects Act.

\section{Competing interests}

SM is principal investigator of the Highlow study (Clinicaltrials.gov registration 01828697), supported by a grant from GlaxoSmithKline. The previously performed ALIFE study [8] was supported by a grant (945-27003) from the Netherlands Organization for Health Research and

Development and a grant from GlaxoSmithKline. No other authors have any competing interests.

\section{Authors' contributions}

The ALIFE2 study was designed by PJ, SM and MG. PJ wrote the first version of this manuscript. SM and MG commented on the text and supervised the writing of the manuscript. SQ leads the funding applications for the study for the UK. BH commented on the statistical analyses sections. SQ, KB, BB, JB, AC, MD, MdS, OH, AH, KJ, CK, WK, BM, HT, HS, MS, HV, JV and JdV are all involved in the ALIFE2 study as investigators. All authors read and approved the final manuscript.

\section{Authors' information}

SM and MG were principal investigators of the ALIFE study [8].

\section{Acknowledgements}

The ALIFE2 study is funded in part by a VIDI innovative research grant for SM, The Netherlands Organization for Health Research and Development (ZonMw, 016.126.364).

A Research for Patient Benefit grant was approved by the National Institute for Health Research.

Study funders have no role in or authority over the study design, data collection, management, analyses, writing of the report or publication decisions.

We would like to thank all (international) colleagues for their critical input on the design of the ALIFE2 study. We would further especially like to thank Tessa de Vries, research nurse AMC, for her help in conducting the study.

\section{Author details}

${ }^{1}$ Department of Vascular Medicine, Academic Medical Center, University of Amsterdam, Meibergdreef 9, 1105 AZ Amsterdam, the Netherlands. Division of Reproductive Health, Warwick Medical School, The University of Warwick, Clifford Bridge Road, Coventry CV2 2DX, UK. ${ }^{3}$ Department of Obstetrics and Gynecology, Leiden University Medical Center, Albinusdreef 2, 2333 ZA Leiden, the Netherlands. "Division Woman, Mother and Child, Tergooiziekenhuis, Rijksstraatweg 1, 1261 AN Blaricum, the Netherlands. ${ }^{5}$ Department of Obstetrics and Gynecology, Jeroen Bosch Ziekenhuis, Henri Dunantstraat 1, 5223 GZ s-Hertogenbosch, the Netherlands. ${ }^{6}$ Department of Gynaecology, Birmingham Women's Hospital and School of Clinical and Experimental Medicine, University of Birmingham, Vincent Drive, Birmingham B15 2TT, UK. ${ }^{7}$ Division of Hematology, CHU Ste-Justine, University of Montreal, 3175 Cote Ste-Catherine, Montreal H3T 1C5, QC, Canada. ${ }^{8}$ Hematology - Medical Oncology Division, Department of Medicine, New York-Presbyterian Hospital/ Weill Cornell Medical College, 1305 York Avenue 7th Floor Room 51, New York, NY 10021, USA. ${ }^{9}$ Department of Obstetrics and Gynecology, Radboud University Medical Center, Geert Grooteplein-Zuid 10, 6525 GA Nijmegen, the Netherlands. ${ }^{10}$ Department of Obstetrics and Gynecology, University Medical Center Groningen, Hanzeplein 1, 9700 RB Groningen, the Netherlands. ${ }^{11}$ Department of Clinical Epidemiology, Biostatistics and Bioinformatics, Academic Medical Center, University of Amsterdam, Meibergdreef 9, 1105 AZ Amsterdam, the 
Netherlands. ${ }^{12}$ Department of Hematology and Hemostasis, Universitair Ziekenhuis Brussel - Vrije Universiteit Brussel, Laarbeeklaan 101, 1090 Brussels, Belgium. ${ }^{13}$ Department of Obstetrics and Gynecology, Máxima Medical Center, De Run 4600, Postbus 7777, 5500MB Veldhoven, the Netherlands. ${ }^{14}$ Department of Obstetrics and Gynecology, Isala Klinieken, Dokter van Heesweg 2, 8025 AB Zwolle, the Netherlands. ${ }^{15}$ The Robinson Institute, School of Paediatrics and Reproductive Health, University of Adelaide, Adelaide 5000, SA, Australia. ${ }^{16}$ Division Woman \& Baby, University Medical Center Utrecht, Postbus 85090, 3508 AB Utrecht, the Netherlands. ${ }^{17}$ Department of Gynecology and Obstetrics at Maastricht University Medical Centre, P Debyelaan 25, 6229 HX Maastricht, the Netherlands. ${ }^{18}$ Department of Obstetrics and Gynecology, University of Illinois College of Medicine, 820 S Wood Street, M/C 808, Chicago, IL, USA. ${ }^{19}$ Department of Obstetrics \& Gynecology, Onze Lieve Vrouwe Gasthuis, Oosterpark 9, 1091 AC Amsterdam, the Netherlands. ${ }^{20}$ Department of Obstetrics, Gynecology and Reproductive Medicine, Amphia Hospital, Langendijk 75, 4819EV Breda, the Netherlands. ${ }^{21}$ Department of Obstetrics, Vrije Universiteit Medical Center, PO Box 7057, 1007 MB Amsterdam, the Netherlands. ${ }^{22}$ Center for Reproductive Medicine, Department of Obstetrics and Gynecology, Academic Medical Center, University of Amsterdam, Meibergdreef 9, 1105 AZ Amsterdam, the Netherlands.

Received: 27 November 2014 Accepted: 13 April 2015

Published online: 07 May 2015

\section{References}

1. Regan L, Rai R. Epidemiology and the medical causes of miscarriage Baillieres Best Pract Res Clin Obstet Gynaecol. 2000;14:839-54.

2. Poland BJ, Miller JR, Jones DC, Trimble BK. Reproductive counseling in patients who have had a spontaneous abortion. Am J Obstet Gynecol. 1977:127:685-91

3. Larsen EC, Christiansen $\mathrm{OB}$, Kolte AM, Macklon N. New insights into mechanisms behind miscarriage. BMC Med. 2013;11:154.

4. Rey E, Kahn SR, David M, Shrier I. Thrombophilic disorders and fetal loss: a meta-analysis. Lancet. 2003;361:901-8.

5. Robertson L, Wu O, Langhorne P, Twaddle S, Clark P, Lowe GD, et al. Thrombophilia in pregnancy: a systematic review. Br J Haematol. 2006:132:171-96.

6. Quenby S, Mountfield S, Cartwright JE, Whitley GS, Chamley L, Vince G. Antiphospholipid antibodies prevent extravillous trophoblast differentiation. Fertil Steril. 2005;83:691-8.

7. de Jong PG, Kaandorp S, Di NM, Goddijn M, Middeldorp S. Aspirin and/or heparin for women with unexplained recurrent miscarriage with or without inherited thrombophilia. Cochrane Database Syst Rev. 2014;7:CD004734.

8. Kaandorp SP, Goddijn M, van der Post JA, Hutten BA, Verhoeve HR, Hamulyak K, et al. Aspirin plus heparin or aspirin alone in women with recurrent miscarriage. N Engl J Med. 2010:362:1586-96.

9. Clark P, Walker ID, Langhorne P, Crichton L, Thomson A, Greaves M, et al SPIN (Scottish Pregnancy Intervention) study: a multicenter, randomized controlled trial of low-molecular-weight heparin and low-dose aspirin in women with recurrent miscarriage. Blood. 2010;115:4162-7.

10. Visser J, Ulander VM, Helmerhorst FM, Lampinen K, Morin-Papunen L, Bloemenkamp KW, et al. Thromboprophylaxis for recurrent miscarriage in women with or without thrombophilia. HABENOX: a randomised multicentre trial. Thromb Haemost. 2011;105:295-301.

11. Goel N, Tuli A, Choudhry R. The role of aspirin versus aspirin and heparin in cases of recurrent abortions with raised anticardiolipin antibodies. Med Sci Monit. 2006:12:CR132-6.

12. Kutteh WH. Antiphospholipid antibody-associated recurrent pregnancy loss: treatment with heparin and low-dose aspirin is superior to low-dose aspirin alone. Am J Obstet Gynecol. 1996;174:1584-9.

13. Rai R, Cohen H, Dave M, Regan L. Randomised controlled trial of aspirin and aspirin plus heparin in pregnant women with recurrent miscarriage associated with phospholipid antibodies (or antiphospholipid antibodies). BMJ. 1997:314:253-7.

14. Farquharson RG, Quenby S, Greaves M. Antiphospholipid syndrome in pregnancy: a randomized, controlled trial of treatment. Obstet Gynecol. 2002;100:408-13.

15. Laskin CA, Spitzer KA, Clark CA, Crowther MR, Ginsberg JS, Hawker GA, et al. Low molecular weight heparin and aspirin for recurrent pregnancy loss: results from the randomized, controlled HepASA Trial. J Rheumatol. 2009;36:279-87.
16. Rodger MA, Hague WM, Kingdom J, Kahn SR, Karovitch A, Sermer M, et al. Antepartum dalteparin versus no antepartum dalteparin for the prevention of pregnancy complications in pregnant women with thrombophilia (TIPPS): a multinational open-label randomised trial. Lancet. 2014:384:1673-83.

17. Royal College of Obstetricians and Gynaecologists: Green-top Guideline No. 17. The investigation and treatment of couples with recurrent first-trimester and second-trimester miscarriage. RCOG 2011.

18. Bates SM, Greer IA, Middeldorp S, Veenstra DL, Prabulos AM, Vandvik PO. VTE, thrombophilia, antithrombotic therapy, and pregnancy: antithrombotic therapy and prevention of thrombosis. 9th ed. American College of Chest Physicians Evidence-Based Clinical Practice Guidelines. Chest. 2012;141:e691S-736.

19. ASRM. Evaluation and treatment of recurrent pregnancy loss: a committee opinion. Fertil Steril. 2012;98:1103-11.

20. ASRM. Definitions of infertility and recurrent pregnancy loss: a committee opinion. Fertil Steril. 2013;99:63.

21. Jauniaux E, Farquharson RG, Christiansen OB, Exalto N. Evidence-based guidelines for the investigation and medical treatment of recurrent miscarriage. Hum Reprod. 2006;21:2216-22.

22. de Jong PG, Goddijn M, Middeldorp S. Antithrombotic therapy for pregnancy loss. Hum Reprod Update. 2013;19:656-73.

23. Brenner B, Bar J, Ellis M, Yarom I, Yohai D, Samueloff A. Effects of enoxaparin on late pregnancy complications and neonatal outcome in women with recurrent pregnancy loss and thrombophilia: results from the Live-Enox study. Fertil Steril. 2005;84:770-3.

24. Linkins LA, Dans AL, Moores LK, Bona R, Davidson BL, Schulman S, et al. Treatment and prevention of heparin-induced thrombocytopenia: antithrombotic therapy and prevention of thrombosis. 9th ed. American College of Chest Physicians Evidence-Based Clinical Practice Guidelines. Chest. 2012;141:e495S-530.

\section{Submit your next manuscript to BioMed Central and take full advantage of:}

- Convenient online submission

- Thorough peer review

- No space constraints or color figure charges

- Immediate publication on acceptance

- Inclusion in PubMed, CAS, Scopus and Google Scholar

- Research which is freely available for redistribution 